\title{
Image Forensics of High Dynamic Range Imaging
}

\author{
Philip. J. Bateman^, Anthony T. S. Ho, and Johann A. Briffa \\ University of Surrey, Department of Computing, \\ Guildford, Surrey, GU2 7XH, UK \\ \{P.Bateman, A.Ho, J.Briffa\}@surrey.ac.uk \\ http://www.surrey.ac.uk/computing/
}

\begin{abstract}
This paper introduces a novel area of research to the Image Forensic field; identifying High Dynamic Range (HDR) digital images. We create a test set of images that are a combination of HDR and standard images of similar scenes. We also propose a scheme to isolate fingerprints of the HDR-induced haloing artifact at "strong" edge positions, and present experimental results in extracting suitable features for a successful SVM-driven classification of edges from HDR and standard images. A majority vote of this output is then utilised to complete a highly accurate classification system.
\end{abstract}

Keywords: Image Forensics, High Dynamic Range Imaging.

\section{Introduction}

In standard 24-bit imaging (one byte per channel), a single pixel can be represented by one of over 1.6 million colours. In real-world environments, however, the depth of colour is significantly larger, meaning that digital images often misrepresent them. Consequently, the images would likely contain under or overexposed regions that degrade texture, detail, and colour. Throughout this paper, we refer to such images as Low Dynamic Range ( $L D R$ ) images. In contrast to LDR images, in High Dynamic Range (HDR) imaging, a set of differently exposed images are combined and processed to create an image with a greater range of luminance between the light and dark areas of an image. This creates a more balanced version of the original image that matches the real-world environment more closely. Figure 1 illustrates one example of the difference between LDR and HDR versions of the same scene.

The quality of HDR processed images has led many manufacturers to use it for enhancing images automatically, directly after they are captured. Popular digital cameras with onboard HDR processing include camera phones such as the Apple iPhone 4 and a wide range of DSLR cameras manufactured by Canon, Sony, Nikon, Casio, Pentax, and many more. Furthermore, since HDR imaging

\footnotetext{
* The author's research is funded by an EPSRC CASE award in collaboration with Charteris Plc.
} 


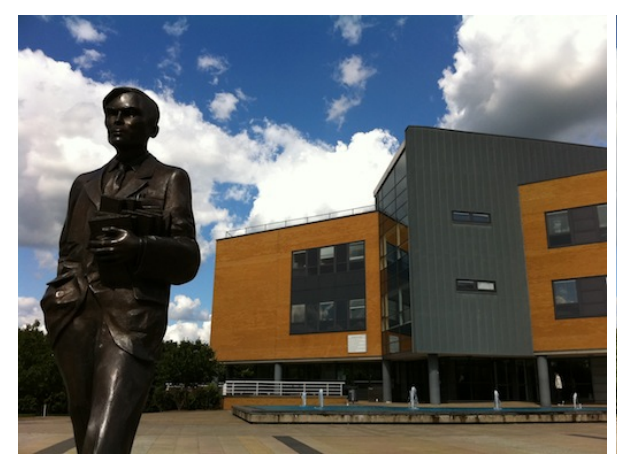

(a) A standard LDR image

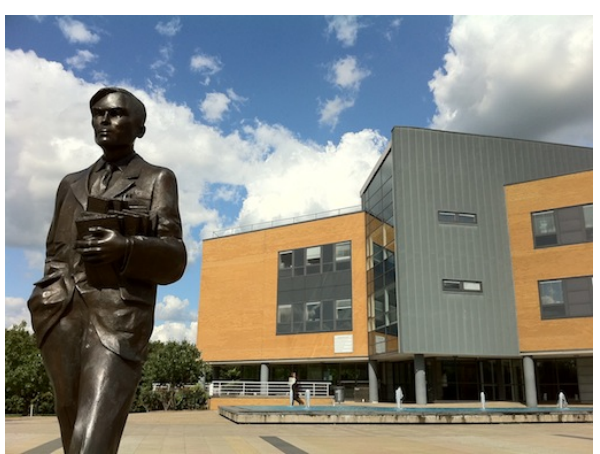

(b) An HDR processed version

Fig. 1. LDR vs. HDR imaging. Note that in the LDR image (a) the dark areas of the statue are under-exposed and therefore lack the depth and detail that is present in the HDR image (b). Similarly, the texture of the clouds are mostly washed out in the LDR image due to over-exposure, where as the HDR version still possesses detail in these regions.

is purely software-driven, the set of camera phone devices increases when we consider downloadable apps available for Android users.

Previously, HDR imaging was considered an image editing tool that was entirely separate from the image acquisition pipeline. Most HDR creations therefore required the use of image editing software such as Adobe Photoshop and GIMP, as well as a bespoke collection of applications such as Photomatix Pro, easyHDR, and DynamicPhoto HDR. However, as an obvious consequence of the increasing availability to HDR imaging, more and more images that appear online are a result of HDR processing. Developing a strategy for the accurate forensic detection of digital images produced from the HDR imaging pipeline is therefore of great importance to ensure we understand the history of a digital image. In this paper, we direct our attention to images captured from HDR devices, with the aim of distinguishing between standard and HDR images taken from the same device. Specifically, this paper discusses and works from the creation of a test set of images captured from the Apple iPhone 4. This device is consistently reported by imaging-hosting website, Flickr.com as the most popular device amongst their community of over 60 million users. When we therefore consider the potential frequency of digital images currently in circulation that originate from this device, it is important to consider it for forensic experiment. Furthermore, the Apple iPhone 4 allows users to capture and process images as HDR natively, meaning it is highly probable that many images in circulation are of this type. We also explore anomalies associated with the HDR imaging pipeline, and combine the output of a trained Support Vector Machine (SVM) with majority voting to classify the images accurately. 
Much of the image forensic research is currently geared towards camera identification. Moreover, the identification of anomalies in the image acquisition pipeline can be used to intrinsically link digital images to their source device. Some of the more acclaimed approaches for achieving this include the analysis of sensor noise patterns [1], radial distortions caused by lens misalignments [2], the affect of the Colour Filter Array (CFA) on pixel colours [3], [4], [5], and the creation of a 34 featured SVM classifier extracted from a range of image properties [6]. Since some manufacturers' allow the direct processing of HDR imaging, it can be considered a possible extension to the image acquisition pipeline, providing a wider range of potential features to aid camera identification. The HDR imaging pipeline is complicated and varies with the implementation. It is therefore likely that fingerprints of individual manufacturer implementations of HDR imaging can directly aid camera identification. Currently, we believe that there is no literature related to Image Forensics for solving the HDR vs. standard problem. It seems logical to suggest that this area will grow in popularity based on the growing trend of applications created for producing HDR images.

This paper introduces the key steps in creating an HDR image, before defining the usage of Homomorphic Filtering for compressing the dynamic range to one that is compatible with output devices. A resulting anomaly from this process is then highlighted, before presenting our strategy for classifying HDR and LDR images. Our experimental results are followed by a concluding summary of our work and potential paths for future work.

\section{High Dynamic Range Imaging Pipeline}

The High Dynamic Range imaging pipeline is a complicated mixture of many processes, sub-processes, and bespoke strategies. Each implementation of HDR imaging can create output images that are significantly different from each other. For example, some implementations seek to produce artistic images that appear as though they have been heavily post-processed, whereas other implementations aim to produce more natural-looking images that closely match real-world scenes. Even when multiple separate implementations share the same desired output, there will still exist some subtle differences that can be useful for forensic analysis.

In Figure 2 we present a simplified model of the HDR pipeline that encompasses the main activities. The figure shows that the dynamic range of a realworld scene is much larger than that of a digital camera. Consequently, when a digital camera captures the scene, a lot of detail in the bright and dark regions is lost in over and under-exposed regions. In contrast, HDR imaging solves this problem by capturing a minimum of two differently exposed images that capture the detail in the highlights and shadows of the scene, as well as the main focal point. These images are then combined to produce a single HDR image with a dynamic range much closer to the real-world scene. However, since display devices and printers have a much smaller dynamic range, the image must be compressed to remove insignificant luminance data so that it can be viewed or 
printed. This process is known as Tone Mapping, and has been the focus of much HDR research in the past few years [7], [8], [9], [10]. The main challenge lies in the fact that there is no universal solution to the tone-mapping problem, since it depends on the scene captured as to how the dynamic range can be reduced without damaging the natural feel of the image.

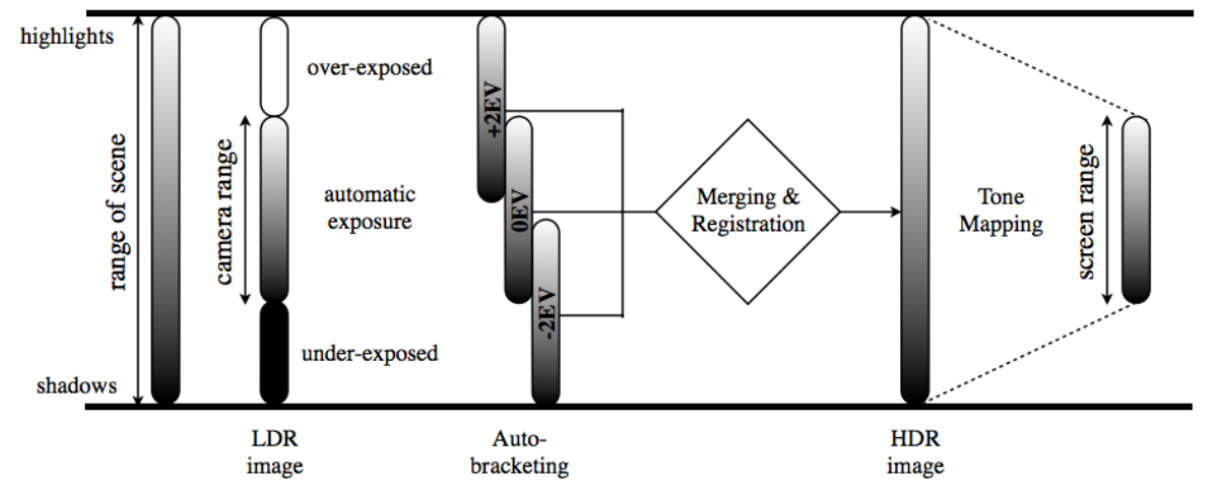

Fig. 2. The HDR imaging pipeline (adapted from [12]).

Oppenheim et al. were amongst the first authors to formally introduce this dilemma, and presented several ideas for tone mapping that are present in many of today's tone mapping operators [11]. Arguably the most dominant of these ideas was the use of Homomorphic Filtering for frequency-dependant compression of luminance components. This concept is discussed in the following section.

\section{$3 \quad$ Homomorphic Filtering}

It is well documented in image processing literature that a given image is comprised of two respective brightness and contrast components: illuminance and reflectance, and that the human brain uses these components to complete the scenes that our eyes capture [13]. The illuminance component refers to the available light that radiates the scene, and reflectance refers to the light that is reflected off of the various objects that the scene contains. For example, a lake will reflect more light than a park bench (reflectance), but both could be lit by the sun (illuminance). A digital image can therefore be expressed in simplified luminance terms as the product of illuminance and reflectance components:

$$
L_{(x, y)}=i_{(x, y)} \cdot r_{(x, y)} .
$$

where $L_{(x, y)}$ refers to a digital image expressed in luminance terms, and $i_{(x, y)}, r_{(x, y)}$ refer to the illuminance and reflectance components respectively. In 
[11], the authors observe that the illuminance component contains significant redundancy, and can be compressed with a minimal impact to the detail and contrast of the image. Since luminance is a multiplicative product of $i$ and $r$, the components must be separated such that dynamic range compression may be performed on $i$ only. This is achieved by firstly computing the luminance data in terms of the logarithmic space such that:

$$
\log \left(L_{(x, y)}\right)=\log \left(i_{(x, y)}\right)+\log \left(r_{(x, y)}\right) .
$$

Note how the luminance data is now expressed as an additive mix of $i$ and $r$. The authors in [11] state that if the data are further expressed according to the Discrete Fourier Transform (DFT), then low frequencies can be associated with the illuminance component, and high frequencies with the reflectance component. The final task is to apply Homomorphic Filtering to attenuate the low frequencies whilst preserving the high frequencies in order to reduce the negligible illuminance data whilst simultaneously increasing the contrast of the image.

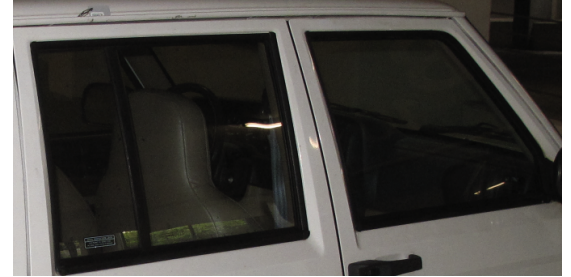

(a) Before Homomorphic Filtering

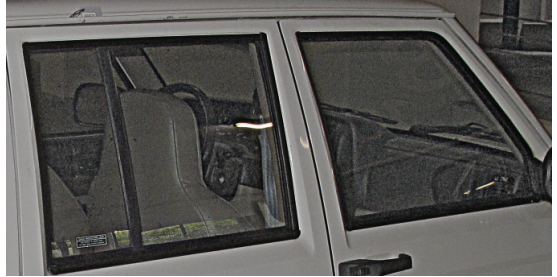

(b) After Homomorphic Filtering

Fig. 3. The effect of Homomorphic Filtering [14]. In the original image image (a) the contrast range is so low that the interior of the vehicle appears dark and lacks detail. However, in the homomorphic filtered image (b) the contrast associated with the detailed regions is emphasised.

Dynamic range compression by Homomorphic Filtering is a popular methodology amongst recent HDR imaging implementations since it is very effective at producing dynamically compressed images with natural-looking end results [15]. However, depending on the contrast difference of two objects, and indeed the attenuation function used, it is common for the filtering process to produce a halo artifact, where objects appear to glow against the background. This artifact is most apparent in edge pixels, since they are a combination of both high and low frequencies, and only the low frequencies are attenuated. The degree to which this artifact is apparent depends on the specific implementation, but in forensic terms this makes it a useful source for fingerprinting. It is this homomorphic filtering artifact that this paper uses for classifying whether an image is HDR or LDR. 


\section{Evaluation Methodology}

In this paper, we hypothesise that digital images will exhibit signs of the halo artifact if they are composed from the HDR imaging pipeline. Similarly, the artifact will not exist to the same magnitude for standard images produced by conventional photography. The identification of haloed regions should therefore help to distinguish HDR and LDR images accurately.

\subsection{Test Strategy}

In our initial work, we establish a library of 100 'landscape' images that are an equal combination of HDR and LDR images taken with the same device (Apple iPhone 4 running iOS 4.3.3). The images are captured with the native camera application, and the device is mounted to a tripod to ensure that the alignment between the HDR and LDR versions of the same scene is consistent. For each image, 'strong' vertical edge points - such as where bright light from the sky merges with a dark object - are extracted and data from these regions are tested to isolate the halo artifact. As discussed in Section 3, the halo artifact is most evident at these such edge regions, as they are comprised of both high and low frequency data.

Following on from a system we have designed to isolate strong edges, we propose the extraction of a frame of pixel data (obtained from the left and right of these edge points) for 100 edges from each image. We then use this data in conjunction with the LibSVM classifier to predict the likelihood that a given edge is an HDR edge. Based on the results of this classification, we then employ majority voting to establish the generalised accuracy for detecting an HDR image.

Figure 4 illustrates a typical example of the degree of haloing associated with LDR and HDR images captured from the Apple iPhone 4. The HDR image shows clear edge anomalies around the strong edge points compared to the equivalent LDR image. What we see is a single bright pixel that separates the boundary of the object with the bright sky background. This is a strong indication that homomorphic filtering has taken place at these two contrasting points, and subsequently a useful fingerprint for detecting HDR imaging.

\subsection{Edge Selection}

The successful extraction of strong edges is key to identifying the halo artifact that forms the basis of our classification process. In essence, we need to establish some automated scheme for extracting the most suitable edges to be considered for evaluation. Figure 5 presents a block diagram of our initial schema for 'Strong Edge' extraction.

Canny Edge Detection. We start by extracting the luminance component of the image, and applying Canny edge detection to find as many edges as possible. 


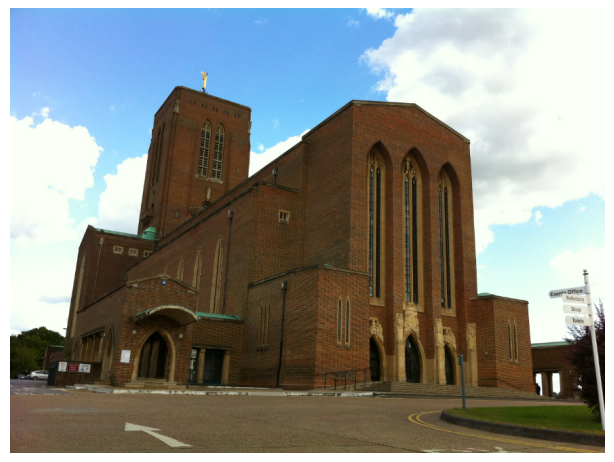

(a) LDR version

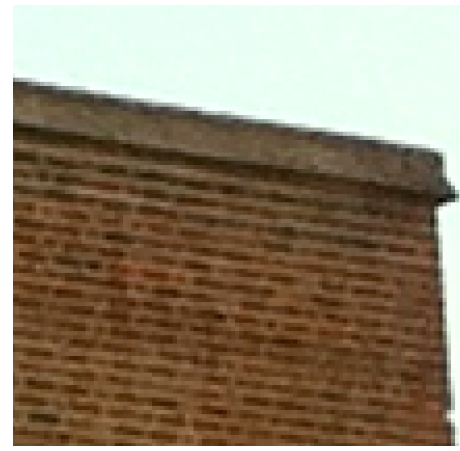

(c) LDR 'strong' edges (magnified)

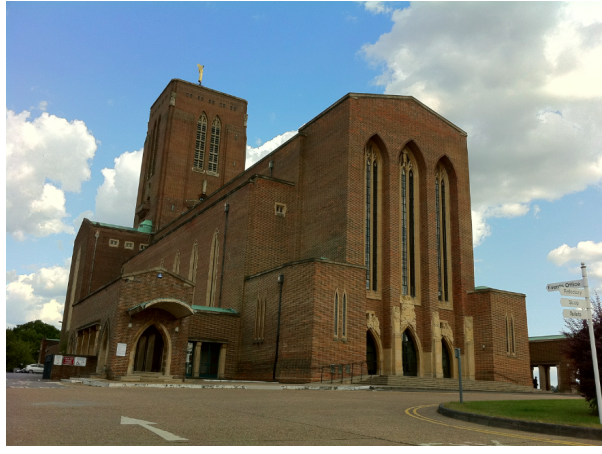

(b) HDR version

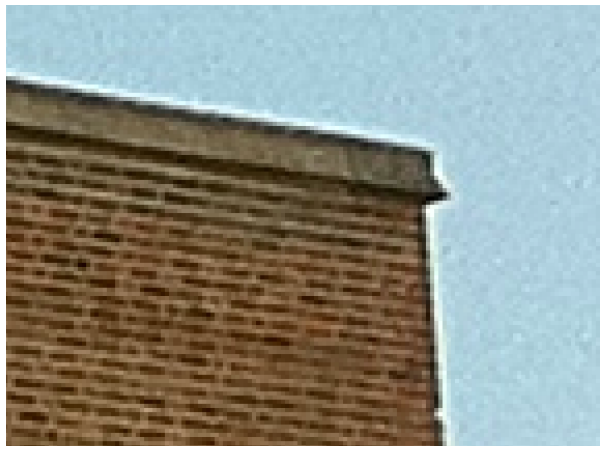

(d) HDR 'strong' edges (magnified)

Fig. 4. HDR and LDR images captured from Apple iPhone 4, and their respective 'strong' edges. Note that whilst the edges of the LDR image (c) do not show visible signs of haloing, the haloing is abundantly obvious for the corresponding edges in the HDR image (d).

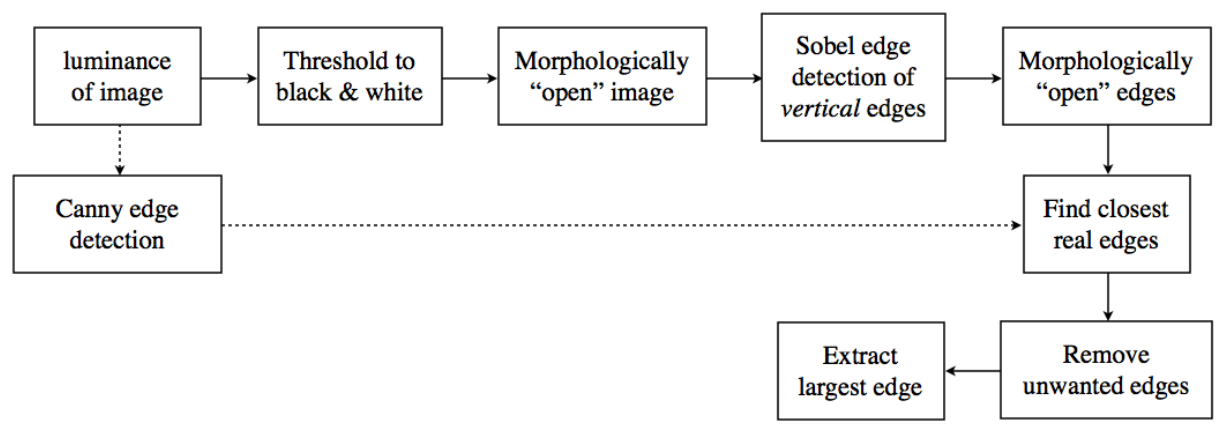

Fig. 5. The 'Strong Edge' extraction process. 
Canny edge detection is the preferred choice as our preliminary testing showed that this operator extracted a higher frequency of the most relevant edges. Figure 6 illustrates how the Sobel, Roberts, and Prewitt operators misses important edges when the brightness of an object closely matches that of the background. In particular, note how the Canny edge operator provides more consistent edge information for the "Refectory" and "Shop" signposts, with respect to other operators. However, since the output of the canny edge detection over-detects many edges from detailed regions, the information must be reduced in order to locate a single edge of interest. To achieve this, the image itself must first undergo several transformations, as discussed in the next section.

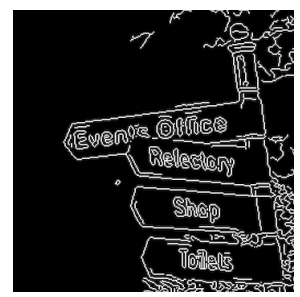

(a) Canny

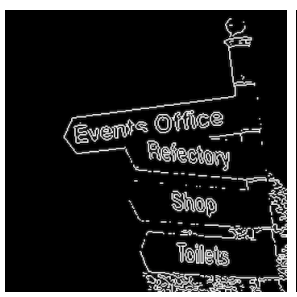

(b) Sobel

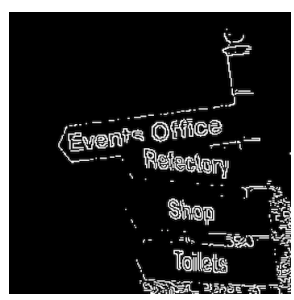

(c) Roberts

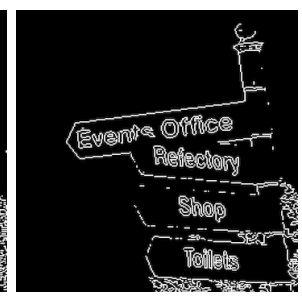

(d) Prewitt

Fig. 6. Edge detection using different operators, each with perceptually optimum threshold values.

\section{$5 \quad$ Experimental Results}

Threshold to Binary Form. In order to reduce the amount of edges that are detected, the detailed regions of the image must be reduced such that all that remains is the edge associated with objects that neighbour significantly contrasting backgrounds. In this model, we firstly threshold the luminance data to produce a binary, black and white pixel representation. The "open" morphological operator is then used to merge the detailed regions in the black and white regions.

Edge Reduction. Since this initial work focuses only on vertical edges, we can apply Sobel edge detection on the opened image to extract only the edges that are likely to be of interest. Now that the detail has been removed from the image, we obtain a much smaller number of edges than we obtained from the Canny edge detection phase. However, due to the fact that morphological opening is a combination of erosion and dilation, the Sobel edges we extract may be slightly misaligned with the original image. We therefore consider the Sobel edges an estimate of interesting edges, and obtain the closest neighbouring edges 
from the Canny edge image. The remaining connected edges are then iteratively processed to determine whether or not they satisfy certain conditions. Assuming for this example that the object is expressed as pixel value 0 , and the background is expressed as 1 , then the conditions are:

- mode of 5 pixels left of edge $=0$.

- mode of 5 pixels right of edge $=1$.

- $\mid$ angle of connected edge $\mid \leq 30^{\circ}$ of vertical axis.

The first rule checks that the object is on the left, while the second rule also checks that the background is on the right of the connected edges. The third rule checks that the edge is close to vertical. The haloing artifact exists as a normal vector to the edge. To simplify the scheme, we consider the haloing artifact that is a normal vector of the vertical axis such that a horizontal extraction of pixel data would encapsulate the full artifact. If the connected edge were at $45^{\circ}$, for example, then we would need to consider and evaluate diagonal pixels. When a connected edge does not satisfy all of the conditions, they will be dropped, and only the edges that are likely to yield the most useful results remain. Figure 7 illustrates several examples of the selected edges that will be used to isolate signs of the haloing artifact.

\section{Experimental Results}

\subsection{Pixel Distribution}

Using the edge selection scheme discussed in Section 5, we obtain the selected edges for each individual image. We then extract a horizontal frame of pixel data centred on each individual edge position, that describes the distribution of pixel data for the object (left) and background (right) that neighbour the edge. Figure 8 illustrates the typical pixel distribution extracted from LDR and HDR images, where a frame radius of 128 pixels is selected.

The vertical line at zero represents the location of the edge pixel. Of particular interest is the distribution of the pixel data that immediately follows the edge. For the LDR image, there is a small peak where the pixels are seemingly slightly brighter than the remainder of the data. In comparison, this peak is much larger for HDR images where it is noted that the pixels immediately right of the edge are more contrasting to the remainder of the data. This is the result of the HDR version containing more colour and texture data than its LDR counterpart, and a clear graphical representation of what was noted from Figure 4 .

\subsection{Classification of HDR vs. LDR Edges}

The plots illustrated in Figure 8 are actually a fair representation of the plots obtained for each of the images. In each case, the LDR version shows a smaller peak when compared to the equivalent HDR image. This provides further confidence that extracting the correct features from this data could help to distinguish 

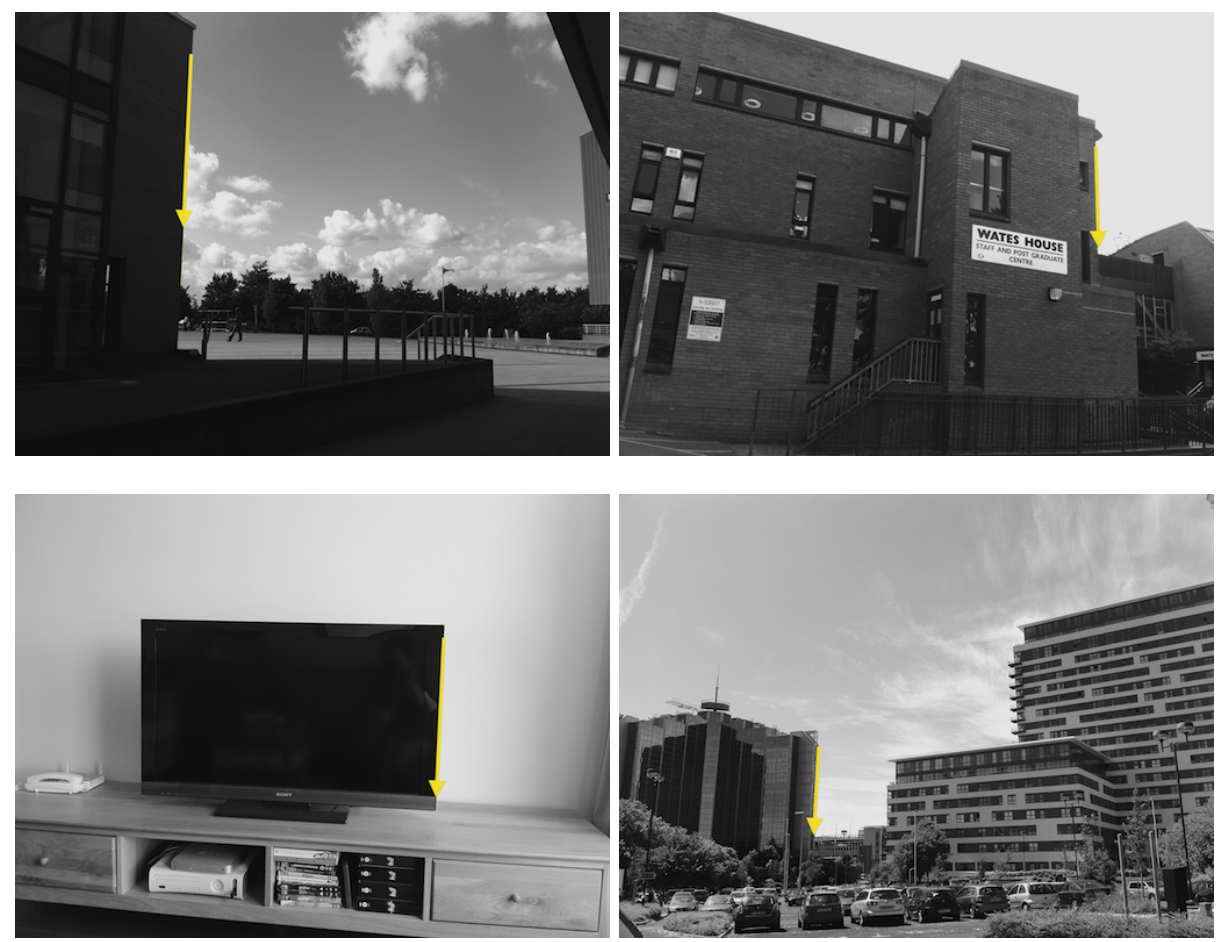

Fig. 7. Examples of edges extracted from the Edge Selection process. 


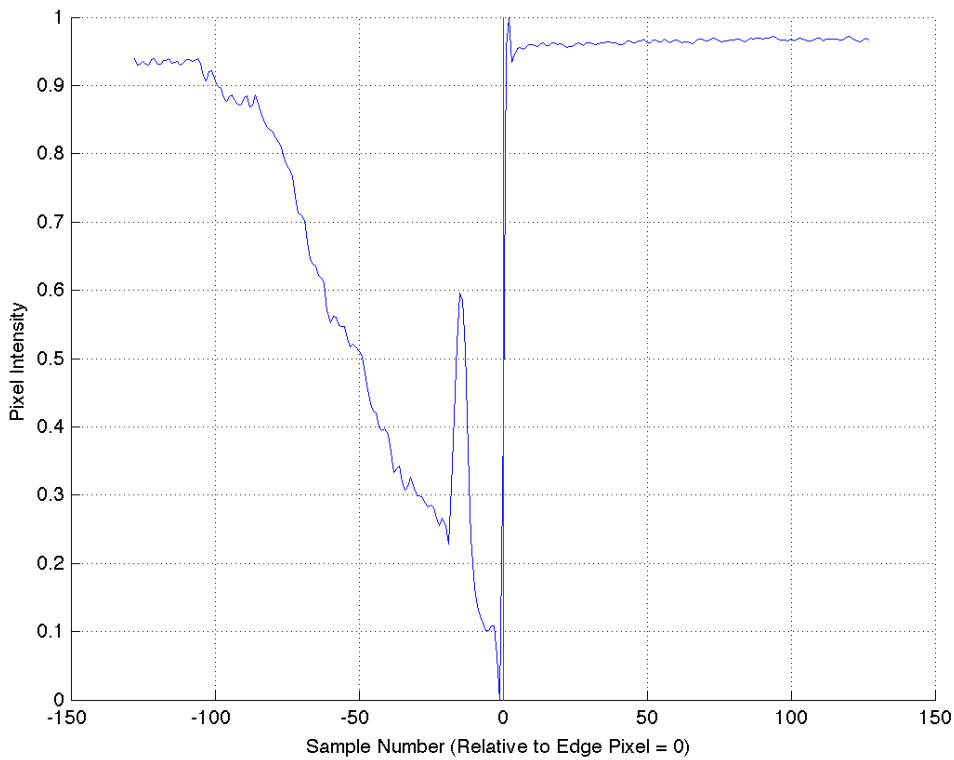

(a) Pixel distribution from HDR Image

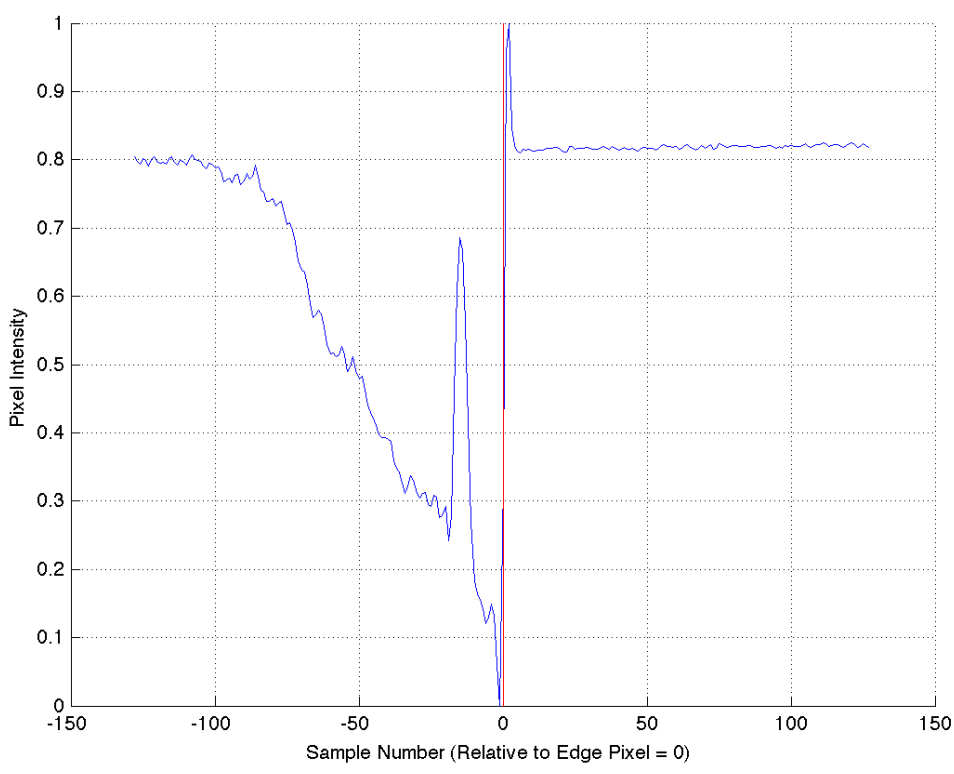

(b) Pixel distribution from LDR Image

Fig. 8. The pixel distribution of LDR (a) and HDR (b) edges. The data has been normalised to intensity values for clearer interpretation. 
between both image types. Of course, the magnitude to which this peak extends is proportionate to the image pixels. If the background is relatively white (for example, in a cloudy scene) then the peak will be smaller than if the sky is a deep blue. However, since it is unlikely that the distribution of pixel data in white clouds will exactly equal the intensity of the halo, a peak always exists. For this reason, it is arguably inappropriate to base feature extraction in the spatial domain. However, accepting that the peak following the edge pixel exists as a common characteristic between the images, it is likely that a DFT transformation will emphasise this trait. We therefore convert each extracted frame of pixels to the DFT domain. By randomly selecting 100 edges from the edge selection process, we sample the pixel data and obtain the magnitude of DFT for each. We then reduce the DFT output to half the original length by removing the symmetric data. Using simply this output, we create a training data set for LibSVM classification. The training set is comprised of 100 edges from $90 \%$ of the images. More precisely, the training set will be a $9000 \times 128$ data matrix, since 90 of the 100 images are used for training, and a 1x128 DFT vector is computed for 100 edges for each image. The remaining 10 images are processed in exactly the same way and are used as test data for the LibSVM classifier.

\subsection{Results}

The LibSVM classifier was able to classify each individual edge with an overall accuracy of $85.1 \%$. Considering that we use 100 edges from every image, this preliminary result provides further proof that the proposed use of the halo artifact is consistent for both image types. However, this classification alone does not demonstrate the success as to whether an entire image is classified as either LDR or HDR. To achieve this, the output of the data classification must be aggregated to groups of 100, where each group represents all the edges for the same image. Using a majority voting strategy, the individual images can be classified with respective levels of confidence. Table 6.3 shows the 'actual' class type (LDR or HDR) for the 10 images tested. The majority voting process correctly predicts the class type in each instance leading to a generalised classification accuracy of $100 \%$. For each image, the percentage of correctly classified edges is computed to provide a confidence level to which each classification is made.

Since the majority voting process implies an accuracy of $100 \%$, we have proved that our scheme can accurately use a single property of HDR imaging to distinguish between LDR and HDR images. The individual levels of confidence from each of these classifications is as high as $100 \%$, but also decreased to $55 \%$. After a brief evaluation of the image this data corresponds to, it is clear that the image is highly textured, and this negatively affects our scheme for extracting strong edges. In this example, a weaker edge was selected, and the data corresponding to the halo was not captured fully. The requirement of developing a more precise scheme is therefore of interest to future work.

To check for manufacturer-specific consistencies in the halo artifact, a small number of random HDR and LDR images have been collected from 2 other Apple iPhone 4 devices. For each of the images tested, the halo artifact exists to the 


\begin{tabular}{|c|c|c|c|}
\hline Test Image & Actual & Predicted & Accuracy (\%) \\
\hline 1 & HDR & HDR $\boldsymbol{v}$ & 88 \\
2 & HDR & HDR $\boldsymbol{v}$ & 99 \\
3 & HDR & HDR $\boldsymbol{v}$ & 80 \\
4 & HDR & HDR $\boldsymbol{v}$ & 69 \\
5 & HDR & HDR $\boldsymbol{v}$ & 55 \\
6 & LDR & LDR $\boldsymbol{v}$ & 87 \\
7 & LDR & LDR $\boldsymbol{v}$ & 92 \\
8 & LDR & LDR $\boldsymbol{V}$ & 100 \\
9 & LDR & LDR $\boldsymbol{v}$ & 91 \\
10 & LDR & LDR $\boldsymbol{v}$ & 90 \\
\hline
\end{tabular}

Table 1. Final classification of LDR vs. HDR images, and the respective confidence levels.

same degree, and similar classification accuracies were obtained. Furthermore, the latest iOS software beta 5.0 was installed to a 4th Apple iPhone 4 device to ensure that the halo artifact has not been addressed by a software update distributed by the manufacturer. Again, the halo artifact was still notably present in HDR images.

It is expected that each manufacturer-driven implementation of the HDR imaging pipeline will inherit anomalies that can be traced to the device in much the same way that camera identification research functions. Furthermore, HDRinduced anomalies such as the haloing artifact are likely to be handled differently depending on the implementation. Since there are many applications available for mobile devices to produce HDR images, it is therefore feasible that the specific application used to create them can be traced.

\section{Conclusion}

In this paper, we have introduced a novel area for image forensic research, and proposed a forensic scheme for identifying the halo artifact induced by HDR imaging, in images collected from the Apple iPhone 4. The scheme is capable of extracting the most suitable edges for analysis, converting them to an appropriate feature representation, and classifying the image successfully. We have presented a proof of concept by presenting our initial experiments and methodology for correctly classifying LDR and HDR images with a $100 \%$ success rate on a small test set. Of interest to future work is the obvious requirement of improving the confidence of individual classifications; most logically by refining the edge selection scheme. Our current strategy for identifying the halo artifact functions only on vertical edges, but can be expanded for horizontal edges in order to strengthen the likelihood that the extracted edge data captures haloed regions. Beyond this, a system for processing any edge could be engineered such that the frame data is extracted as a normal vector to the edge direction. These 
modifications would enable the scheme to be more compatible with a wider range of scenes.

\section{Acknowledgements}

The authors would like to extend thanks and gratitude to Charteris Plc. for supporting this research.

\section{References}

1. J. Lukáš, J. Fridrich, and M. Goljan, "Digital Camera Identification From Sensor Pattern Noise," IEEE Transactions on Information Security and Forensics, vol. 1(2), pp. 205-214, 2006.

2. K. S. Choi, E. Y. Lam, and K. K. Y. Wong, "Source Camera Identification Using Footprints From Lens Aberration," Proceedings of the SPIE, vol. 6069, pp. 172-179, 2006.

3. S. Bayram, H. T. Sencar, N. Memon, and I. Avcibas, "Source Camera Identification Based on CFA Interpolation," Proceedings of IEEE ICIP, vol. 3, pp. 69-72, 2005.

4. O. Celiktutan, I. Avcibas, B. Sankur, and N. Memon, "Source Cell-phone Identification," IEEE Signal Processing and Communications Applications, pp. 1-3, 2005.

5. Y. Long and Y. Huang, "Image Based Source Camera Identification using Demosaicking," IEEE 8th Workshop on Multimedia Signal Processing, pp. 419-424, 2006.

6. M. Kharrazi, H. T. Sencar, and N. Memon, "Blind Source Camera Identification," International Conference on Image Processing, vol. 1, pp. 709-712, 2004.

7. R. Fattal, D. Lischinski, and M. Werman, "Gradient domain high dynamic range compression," Proceedings of the $29^{\text {th }}$ Annual Conference on Computer Graphics and Interactive Techniques, vol. 21, pp. 249-256, 2002.

8. R. Mantiuk, K. Myszkowski, and H. P. Seidel, "A Perceptual Framework for Contrast Processing of High Dynamic Range Images," In ACM Transactions on Applied Perception, vol. 3, pp. 286-308, 2006.

9. G. Krawczyk, K. Myszkowski, Karol and H. P. Seidel, "Computational Model of Lightness Perception in High Dynamic Range Imaging," Human Vision and Electronic Imaging XI, ISEST/SPIE's $18^{\text {th }}$ Annual Symposium on Electronic Imaging, 2006.

10. G. Qiu, J. Guan, J. Duan, M. Chen, "Tone Mapping for HDR Image using Optimization A New Closed Form Solution," $18^{\text {th }}$ International Conference on Pattern Recognition, pp. 996-999, 2006.

11. A. V. Oppenheim, R. Schafer, and T. Stockham, "Nonlinear Filtering of Multiplied and Convolved Signals," in Proceedings of the IEEE, 56(8), pp. 12641291, 1968.

12. P. Debevec, Y. Yu and G. D. Borshukov, "Efficient View-Dependent Image-Based Rendering with Projective Texture-Mapping," Eurographics Rendering Workshop pp. 105-116, 1998.

13. R. C. Gonzalez, and R. E. Woods, "Digital Image Processing (third edition)," Pearson Prentice Hall, ISBN: 978-0-13-168728-8, 2007.

14. J. C. Russ, "Forensic Uses of Digital Imaging," CRC Press, ISBN: 978-0-84-930903$8,2001$. 
15. E. Reinhard, G. Ward, S. Pattanaik, and P. Debevec, "High Dynamic Range Imaging: Acquisition, Display, and Image-Based Lighting," Morgan Kauffman, ISBN: 978-0-12-585263-0, 2005. 